\title{
Special issue with selected papers from DISC 2009
}

\author{
Idit Keidar
}

Published online: 28 October 2011

(C) Springer-Verlag 2011

This issue contains five papers selected among the thirty-three presented at the 23rd International Symposium on Distributed Computing (DISC 2009) in Elche, Spain. These five best papers were selected by the Program Committee. They are representative of the high quality research exhibited at DISC and of the topics covered therein. The papers appear here in revised and expanded form.

The first two papers deal with agreement in asynchronous shared memory. This special issue begins with DISC's Best Paper Award winner, The Disagreement Power of an Adversary by Delporte-Gallet, Fauconnier, Guerraoui, and Tielmann. While traditional work in the field assumes that up to some threshold, $t$, of processes may crash, this work considers adversaries that are not necessarily uniform, in the sense that they may crash certain combinations of processes and not others. There are $2^{2^{n}}$ such adversaries. In order to classify them, the paper introduces the notion of disagreement power: the biggest integer $k$ for which the adversary can prevent processes from agreeing on $k$ values. Using this notion, the authors show that there are in fact $n$ equivalence classes of adversaries.

A related paper is $O n$ Set Consensus Numbers by Gafni and Kuznetsov. It considers colorless tasks, in which a process is free to adopt the input or output value of any other participating process. The paper shows that any failure detector that solves a colorless task that cannot be solved $k$-resiliently also solves $k$-set consensus. More generally, it shows that every colorless task $T$ can be characterized by its set consensus number: the largest $k$ in $\{1, \ldots, n\}$ such that $T$ is solvable

I. Keidar $(\varangle)$

Department of Electrical Engineering,

The Technion, Haifa 32000, Israel

e-mail: idish@ee.technion.ac.il $(k-1)$-resiliently. As a corollary, the paper determines the weakest failure detector for solving $k$-set consensus under all possible assumptions on when and where failures might occur.

The next three papers deal with network (message-passing) algorithms. The first among these, Randomization can be a Healer: Consensus with Dynamic Omission Failures by Moniz, Neves, Correia, and Verissimo, won the Best Student Paper Award at DISC. Motivated by wireless ad-hoc networks, the paper considers networks that are prone to dynamic communication failures. It employs randomization to circumvent Santoro and Widmayer's well-known impossibility result for (deterministic) consensus in this model. The paper presents an algorithm that is always safe, and is probabilistically live.

Next appears New Bounds for the Controller Problem by Korman and Emek. This work studies the $(M, W)$-controller, an abstraction for managing the consumption of a global resource in a distributed dynamic network, which has been studied for more than two decades. The paper proves two new lower bounds on its message complexity. It further shows that these bounds are tight using a new controller construction.

The issue concludes with The Abstract MAC Layer by Kuhn, Lynch, and Newport. The purpose of this work is to provide a unified formal framework for studying algorithms and lower bounds for radio networks. It presents an abstract framework, called the Abstract MAC Layer, which provides reliable local broadcast communication. Timing guarantees in this framework are stated in terms of a collection of abstract delay functions applied to the relevant contention. The authors exemplify the use of the Abstract MAC layer by presenting and analyzing two algorithms for the new Multi-Message Broadcast problem. 
I am grateful to the authors for writing these papers and revising them to meet the journal's standards, to the DISC 2009 Program Committee for initially reviewing and selecting the best papers, and to the referees who reviewed them for Distributed Computing. I am also thankful to Hagit Attiya for fostering the collaboration between DISC and Distributed Computing that made this special issue possible.

Idit Keidar

Program Committee Chair, DISC 2009

Guest Editor 